\title{
Aerosol assisted chemical vapour deposited (AACVD) of TiO2 thin film as compact layer for dye-sensitised solar cell
}

\begin{abstract}
Compact $\mathrm{TiO} 2$ has been introduced onto the surface of an indium tin oxide glass slide (ITO), using an aerosol-assisted chemical vapour deposition method. This serves as a blocking layer for a dye-sensitised solar cell (DSSC). The thickness of the compact TiO2 could be controlled by deposition time. X-ray diffraction and Raman spectroscopy analyses reveal that the compact $\mathrm{TiO} 2$ is made up of mixed anatase and rutile phases. The field emission scanning electron microscopy image displays a pyramidal morphology of the compact TiO2. A layer of P25 paste was then smeared onto the compact TiO2-modified ITO, using the doctor's blade method. A post-treatment procedure was applied to remove the contaminants from the prepared hybrid film, by immersing in a hydrochloric acid solution. The photoelectrochemical measurements and $\mathrm{J}-\mathrm{V}$ characterisation of the hybrid film show an approximately fourfold increase in photocurrent density generation $(114.22 \mu \mathrm{A} / \mathrm{cm} 2)$, and approximately $25 \%$ enhancement of DSSC conversion efficiency (4.63\%), compared to the acid-treated $\mathrm{P} 25$ paste alone $(3.68 \%)$.
\end{abstract}

Keyword: D. TiO2; AACVD; Chemical vapour deposition 